\title{
AN UNSUPERVISED METHOD BASED ON FIRE INDEX ENHANCEMENT AND GRNN FOR AUTOMATED BURNED AREA MAPPING FROM SINGLE-PERIOD REMOTE SENSING IMAGERY
}

\author{
Qi Zhang ${ }^{1, *}$, Yao Xiao ${ }^{2}$ \\ ${ }^{1}$ School of Remote Sensing and Information Engineering, Wuhan University, Wuhan, China - zhangqi_whursgcm@whu.edu.cn \\ ${ }^{2}$ State Key Laboratory of Information Engineering in Surveying, Mapping and Remote Sensing, Wuhan University, Wuhan, China - \\ xywhu2014@whu.edu.cn
}

Commission III, WG III/1

KEY WORDS: Burning area mapping, Fire index, Adaptive spatial context information, Uncertainty analysis, GRNN

\begin{abstract}
:
In the current situation of frequent forest fires, the study of forest burned area mapping is important. However, there is still room for improvement in the accuracy of existing forest burning area mapping methods. Therefore, in this paper, an unsupervised method based on fire index enhancement and GRNN (General Regression Neural Network) is proposed for automated forest burned area mapping from single-date post-fire remote sensing imagery. The proposed method first uses adaptive spatial context information to enhance the generated fire index to improve its ability to indicate the burned areas. Then the uncertainty analysis is performed on the enhanced fire index to extract reliable burned samples and non-burned samples for subsequent classifier training. Finally, the improved GRNN model considering the spatial correlation of pixels is used as a classifier to binarize the enhanced fire index to generate the final burned area map. Based on two commonly used fire indexes, NBR (Normalized Burn Ratio) and BAI (Burned Area Index), this paper conducts burned area mapping experiments on a post-fire image of a forest area in Inner Mongolia, China to test the effectiveness of the proposed method, and two commonly used threshold methods (Otsu and Kmeans clustering) are also used to conduct burned area mapping based on threshold segmentation of fire index for comparison experiments. The experimental results prove the effectiveness and superiority of the proposed method. The proposed method is unsupervised and automated, so it has high application value and potential under the current situation of frequent forest fires.
\end{abstract}

\section{INTRODUCTION}

Forests are one of the most important natural resources on the earth, and they have irreplaceable value in regulating climate, maintaining ecological balance and many other aspects. However, in recent years, large-scale forest fires have frequently occurred all over the world (Bastarrika et al., 2011). The mapping of forest burned areas helps to understand the severity of forest fires and their spatial and temporal changes, and then helps forest recovery and management after fire events (Roy et al., 2019). Therefore, the study of forest burned area mapping is very important and necessary.

The spectral fire index calculated from the original spectral bands of the image reflects the probability that each pixel in the image belongs to the burned area. Considering the accuracy and efficiency of the mapping, burned area mapping based on the fire index is currently one of the most popular and applicable forest burned area mapping methods (Veraverbeke et al., 2011). For example, Pulvirenti et al. (2020) constructed an automatic processing chain for near real-time mapping of burned forest areas using Sentinel-2 data based on the delta Normalized Burn Ratio (NBR) index and the Normalized Difference Vegetation Index. Engelbrecht et al. (2017) proposed a Normalized Difference Alpha-Angle Index for burned area identification by using multi-polarisation C-band SAR. Liu et al. (2020) proposed a new burned area change detection approach by using Landsat- 8 OLI data based on the fire index and Otsu algorithm. More research on burned area mapping based on fire index can be found in (Chuvieco et al., 2002; Roteta et al., 2019; Woźniak et al., 2019).

The mapping of the burned areas based on the fire index usually involves two main steps: (1) Calculating the fire index from remote sensing images (2) Binarizing the fire index to generate a burned area map. Generally speaking, in the fire index map, the larger (or the smaller in some indexes) the index value of a pixel, the higher the probability that the pixel belongs to the burned areas. Therefore, after the fire index is generated, most of the existing burned area mapping methods usually use threshold segmentation or clustering to binarize the fire index to generate the final burned area map. In fact, in the fire index map, there are many pixels with high uncertainty, and they have medium index value. It is difficult to accurately determine whether they belong to the burned or non-burned area by simple methods such as threshold segmentation or clustering. Such methods often cause unnecessary missed or false detections, thereby reducing the accuracy and reliability of the burned area mapping. In view of the above considerations, this paper proposes an unsupervised method based on fire index enhancement and GRNN (General Regression Neural Network) for automated forest burned area mapping from single-date post-fire remote sensing imagery.

\footnotetext{
* Corresponding author
} 


\section{PROPOSED METHOD}

As shown in Figure 1, the method proposed in this paper mainly includes four main steps, namely: (1) Fire index calculation from post-fire image; (2) Fire index enhancement based on adaptive spatial context information; (3) Uncertainty analysis to extract burned and non-burned samples; (4) GRNN training and prediction to generate a burned area map.

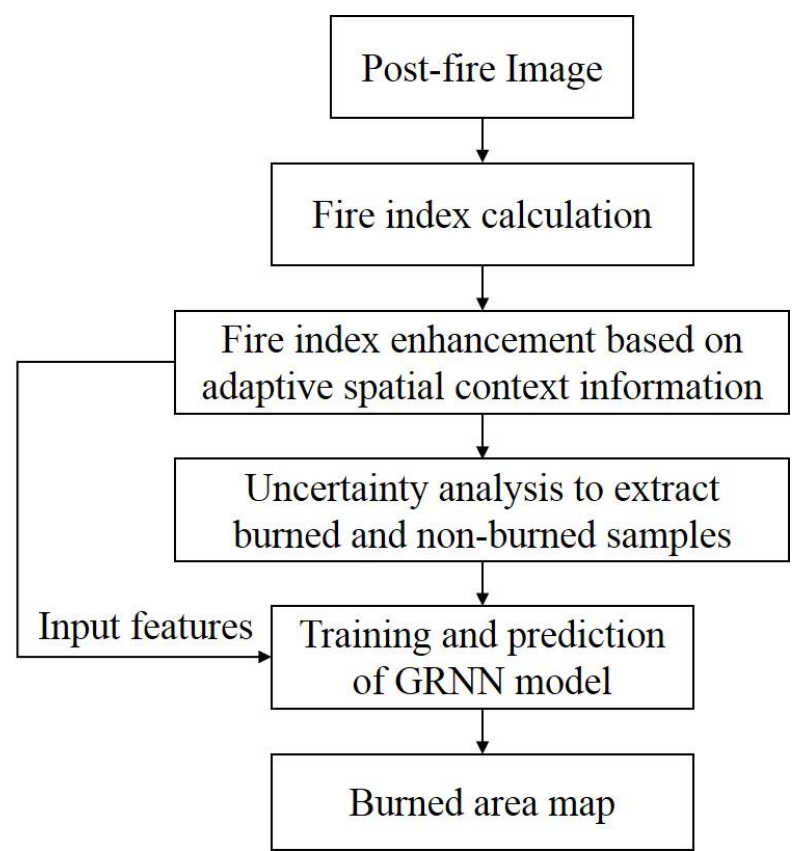

Figure 1. The flow chart of the proposed burned area mapping method

\subsection{Fire Index Calculation from Post-Fire Image}

The first step of the proposed method is to calculate the fire index from the post-fire image. In this study, two commonly used and effective indices, NBR (Veraverbeke et al., 2011) and Burned Area Index (BAI) (Chuvieco et al., 2002) were selected and compared for burned area mapping. The calculation formulas of NBR and BAI are shown in equations (1) and (2).

$$
\begin{aligned}
& N B R=\left(\rho_{\text {NIR }}-\rho_{\text {SWIR }}\right) /\left(\rho_{\text {NIR }}+\rho_{\text {SWIR }}\right) \\
& B A I=\left[\left(\alpha-\rho_{R}\right)^{2}+\left(\beta-\rho_{N I R}\right)^{2}\right]^{-1}
\end{aligned}
$$

where $\rho_{R}, \rho_{\text {NIR }}$, and $\rho_{\text {SWIR }}$ respectively represent the reflectivity of the remote sensing image in the red band, near-infrared (NIR) band, and short-wave infrared (SWIR) band. By referring to related literature (Bastarrika et al., 2011), $\alpha$ and $\beta$ are set to 0.10 and 0.06 , respectively.

NBR uses the law that the spectral reflectance of the burned areas increases in the SWIR band and decreases in the NIR band to separate the burned areas from other ground objects. BAI is constructed based on the distance between the pixel value of the ground object and its reference spectral value, which can emphasize the charcoal signal in the burned areas. NBR and BAI have high sensitivity to fire burning signals, so they are frequently used in research related to forest burned area mapping.

\subsection{Fire Index Enhancement Based on Adaptive Spatial Context Information}

Theoretically, in the fire index map, the larger (or the smaller in some indexes) the index value of a pixel indicates that the pixel is more likely to belong to the burned areas. However, noise or mixed pixels inevitably exist in the original spectral band of the image. Therefore, the fire index map generated directly from the original spectral band of the image cannot accurately reflect the probability that each pixel belongs to the burned areas. For this reason, considering the spatial continuity of the burned area in the image, this paper proposes an index enhancement method based on adaptive spatial context information to enhance the initial fire index map to improve its ability to indicate the burned areas.

Specifically, the proposed enhancement method first uses the region growing algorithm to generate a series of adaptive regions $R_{i}=\left\{R_{i}^{s_{1}}, R_{i}^{s_{2}}, \cdots, R_{i}^{s_{N}}\right\}$ with different scales for each pixel $x_{i}$ in the initial fire index map. Moreover, the series of adaptive regions $R_{i}=\left\{R_{i}^{s_{1}}, R_{i}^{s_{2}}, \cdots, R_{i}^{s_{N}}\right\}$ of each pixel $x_{i}$ are generated at a fixed interval $\Delta s$ with the pixel $x_{i}$ as the initial seed point. $S_{n}$ is the scale parameter, that is, the number of pixels contained in an adaptive spatial region, $n=1,2, \cdots, N$, and $\mathrm{N}$ represents the number of all generated adaptive regions. The interval $\Delta s$ represents the difference between the number of pixels contained in two adaptive regions of adjacent scales, that is, $\Delta s=s_{n+1}-s_{n}$. In this study, $\Delta s$ is set to 5. Obviously, in the process of region growth, when the scale parameter $S_{n}$ is relatively small, as the scale of the adaptive region increases, the number of similar pixels in the region gradually increases, so the homogeneity of the region will gradually increase. When the scale $S_{n}$ increases to a certain extent, as the scale of the adaptive region continues to increase, more heterogeneous information (such as noise, heterogeneous pixels, etc.) will be continuously introduced into the region, so the homogeneity of the adaptive region will gradually decrease. Therefore, in a series of adaptive regions $R_{i}=\left\{R_{i}^{s_{1}}, R_{i}^{s_{2}}, \cdots, R_{i}^{s_{N}}\right\}$ centered on the pixel $x_{i}$, there must be an optimal adaptive region $\tilde{R}_{i}^{s_{n}}$ corresponding to the optimal scale $\tilde{s}_{n}$, which has the highest homogeneity.

In statistics, the Coefficient of Variation (CV) is a commonly used statistical indicator to measure the degree of dispersion of a set of data. It is defined as the ratio of the standard deviation to the mean of this set of data. A large CV indicates that the degree of dispersion of the data is also large, and vice versa. In this section, $\mathrm{CV}$ is used to find the optimal adaptive region $\tilde{R}_{i}^{s_{n}}$ from a series of adaptive regions $R_{i}=\left\{R_{i}^{s_{1}}, R_{i}^{s_{2}}, \cdots, R_{i}^{s_{N}}\right\}$. When the generated adaptive region contains more heterogeneous components, the difference between the pixels contained in the region will be greater, and therefore the corresponding $\mathrm{CV}$ will be greater. Conversely, the stronger the homogeneity of the adaptive region, the smaller the corresponding CV. Therefore, we can conclude that the best optimal adaptive region with the highest homogeneity has the 
smallest $\mathrm{CV}$, and vice versa. According to the definition of $\mathrm{CV}$, the $\mathrm{CV}$ corresponding to an adaptive spatial region $R_{i}^{s_{n}}$ in $R_{i}=\left\{R_{i}^{s_{1}}, R_{i}^{s_{2}}, \cdots, R_{i}^{s_{N}}\right\}$ can be calculated by formula (3).

$$
C V\left(R_{i}^{s_{n}}\right)=\frac{\delta\left(R_{i}^{s_{n}}\right)}{\mu\left(R_{i}^{s_{n}}\right)}
$$

where $C V\left(R_{i}^{s_{n}}\right)$ represents the $\mathrm{CV}$ of the adaptive spatial region $R_{i}^{s_{n}} \cdot \delta\left(R_{i}^{s_{n}}\right)$ and $\mu\left(R_{i}^{s_{n}}\right)$ respectively represent the standard deviation and mean value of the fire index values of all pixels in the adaptive region $R_{i}^{s_{n}}$.

After obtaining the CVs corresponding to all the adaptive regions in $R_{i}=\left\{R_{i}^{s_{1}}, R_{i}^{s_{2}}, \cdots, R_{i}^{s_{N}}\right\}$, according to the principle that the optimal adaptive region has the smallest $\mathrm{CV}$, we can select the optimal adaptive region $\tilde{R}_{i}^{s_{n}}$ corresponding to pixel $x_{i}$.

After that, we spatially enhance the fire index of each pixel based on its optimal adaptive region. Specifically, the enhanced fire index value of each pixel can be calculated according to equation (4).

$$
E I\left(x_{i}\right)=\frac{1}{\tilde{S}_{n}} \sum_{x_{j} \in \tilde{R}_{i}^{s_{n}}} I\left(x_{j}\right)
$$

where $E I\left(x_{i}\right)$ represents the enhanced fire index value of pixel $x_{i}$, and $I\left(x_{j}\right)$ represents the initial fire index value of any pixel $x_{j}$ in the optimal adaptive region $\tilde{R}_{i}^{s_{n}}$ of pixel $x_{i}$ before enhancement. $\tilde{S}_{n}$ represents the scale parameter corresponding to $\tilde{R}_{i}^{s_{n}}$, that is, the total number of pixels contained in $\tilde{R}_{i}^{s_{n}}$.

After this step, an enhanced fire index map can be generated, which can more accurately reflect the probability of each pixel belonging to the burned areas.

\subsection{Uncertainty Analysis to Extract Burned and Non- Burned Samples}

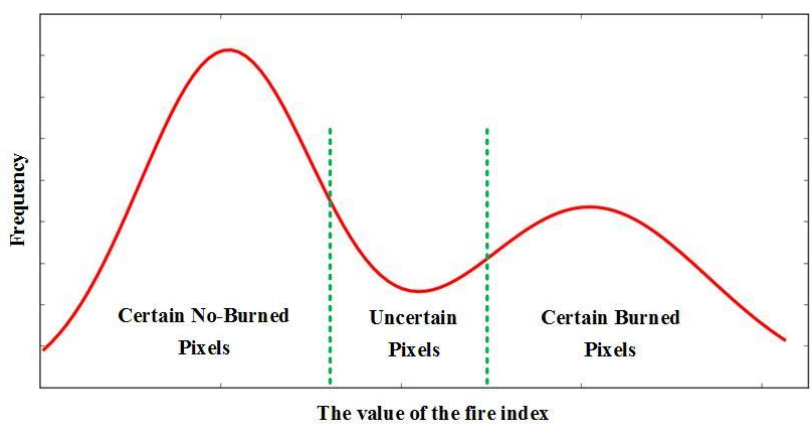

Figure 2. Uncertainty analysis of enhanced fire index.

In the generated enhanced fire index map, the larger (or smaller in some cases) the index value of a pixel, the greater the probability that the pixel belongs to the burned areas. As shown in Figure 2, in the fire index map, there are many pixels with high uncertainty, and they have medium index values. It is difficult to accurately determine whether these uncertain pixels belong to the burned or non-burned areas, so they are one of the main sources of error in burned area mapping. In this subsection, our goal is to identify uncertain pixels and certain pixels (including certain burned pixels and certain non-burned pixels) from the generated enhanced fire index map through uncertainty analysis. Here, the uncertainty analysis based on Fuzzy C-means (FCM) clustering was performed on the enhanced fire index to achieve this goal. FCM clustering is one of the most popular fuzzy clustering algorithms. Unlike traditional hard clustering, which strictly divides each element into a class, FCM clustering regards each cluster as a fuzzy set, and determines the clustering relationship through the membership of the elements. In FCM clustering, each element can belong to different clusters to different degrees at the same time. This fuzzy characteristic of FCM clustering makes it very suitable for uncertainty analysis of the fire index. FCM clustering divides the generated enhanced fire index into three fuzzy clusters by continuously iteratively updating the cluster centers and membership matrix, namely non-burned pixels, uncertain pixels and burned pixels. More details about FCM clustering can be found in (Havens et al., 2012 and Kouhi et al., 2020).

After completing the fuzzy clustering, we can get the membership matrix of the fire index map, which reflects the degree to which each pixel belongs to each class. Then, in accordance with the principle of maximum membership, we can assign each pixel to a specific class, that is, certain non-burned pixels, uncertain pixels or certain burned pixels.

After that, the certain burned pixels and the certain non-burned pixels generated by the uncertainty analysis based on FCM clustering are selected as samples and input into the subsequent classification process because their class information has high reliability.

\subsection{GRNN Training and Prediction to Generate a Burned Area Map}

In some existing burned area mapping methods, the spatial correlation information between adjacent pixels in the image is often not well utilized. In fact, the burned areas in the image has spatial continuity. Therefore, when a pixel is a burned pixel, its adjacent pixels are also likely to be burned pixels. Considering the spatial correlation information of the pixels in the image will help improve the accuracy and reliability of the burned area mapping. To this end, this paper constructs a GRNN considering the spatial correlation information between pixels to binarize the previously generated enhanced fire index to generate the final burned area map.

As shown in Figure 3, the main architecture of the GRNN model used in this paper is consistent with the general GRNN model. It consists of four layers, respectively: input layer, pattern layer, summation layer and output layer. However, in order to make it suitable for burned area mapping and to consider the spatial correlation between pixels in the image, we need to properly modify the input and output of the standard GRNN model. Specifically, for the input of the GRNN model used in this study, we first take each pixel in the enhanced fire index map as the center pixel and extract its $5 * 5$ spatial neighborhood. Then, all pixels in the neighborhood are sorted 
from left to right and top to bottom as shown in Figure 3. Finally, the fire index values of all the sorted pixels are input into the GRNN model as input features. Correspondingly, the number of neurons in the input layer of the GRNN model in this study is 25 . For the output of the GRNN model, what we need is a binarized burned area map, that is, GRNN is required to output the class label of each pixel, which implies whether each pixel is a burned or non-burned pixel. In this study, the class of burned pixels is labeled as 1 while the class of non-burned pixels is labeled as 0 . When the standard GRNN model is used for burned area mapping, its output $\mathrm{Y}$ is a non-binarized value between 0 and 1 . Therefore, $\mathrm{Y}$ needs to be adjusted appropriately. After adjustment, the output $Y^{\prime}$ of GRNN (that is, the final classification label of each pixel) can be generated according to formula (5):

$$
Y^{\prime}=\left\lfloor Y+\frac{1}{2}\right\rfloor
$$

where $\lfloor\cdot\rfloor$ represents the operation of rounding down. That is, when the output result $\mathrm{Y}$ of the original GRNN is greater than or equal to 0.5 , the corresponding pixel belongs to the burned areas, and $Y^{\prime}=1$, otherwise $Y^{\prime}=0$. More details about the internal architecture of GRNN can be found in the literature ( $\mathrm{Li}$ et al., 2011 and Li et al., 2016).

In this step, the modified GRNN model is trained by five-fold cross-validation, and the optimal model is selected for prediction to generate the final burned area map.

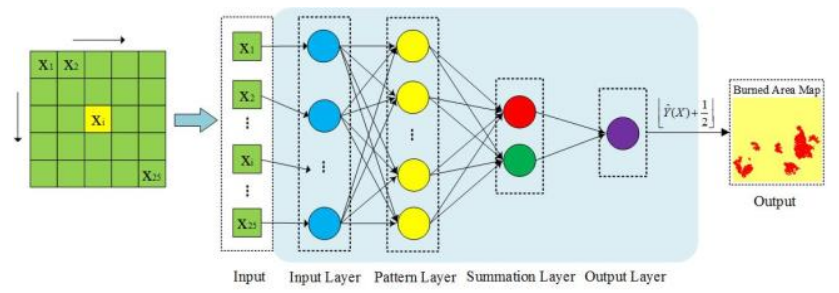

Figure 3. Architecture of the GRNN model used in this study

\section{EXPERIMENTAL RESULTS AND ANALYSIS}

\subsection{Experimental Data and Settings}

To verify the effectiveness of the proposed method, we selected a forest area in Inner Mongolia, China, where a large-scale fire event occurred as the experimental area to conduct burned area mapping experiments. The entire experimental area covers approximately 410 square kilometers. The experimental data is the Landsat image of the forest area after the fire event with a spatial resolution of 30 meters, as shown in Figure 4(a). Figure 4(b) shows the corresponding ground reference map, which was obtained through manual visual interpretation from Landsat images before and after the fire. At the same time, two common burned area mapping methods based on fire index segmentation were also used to map the burned areas for comparison experiments, respectively: Otsu-based threshold segmentation method (Otsu) and Kmeans clustering-based mapping method (Kmeans). In addition, as mentioned earlier, the two commonly used fire indices, NBR and BAI, were used for burned area mapping experiments, and their mapping capabilities were compared. In order to quantitatively compare the mapping performance of different methods, the four commonly used quantitative evaluation indicators: overall accuracy (OA), Kappa coefficient (KC), false alarm rate (FA) and missed alarm rate (MA), are used for mapping accuracy evaluation.

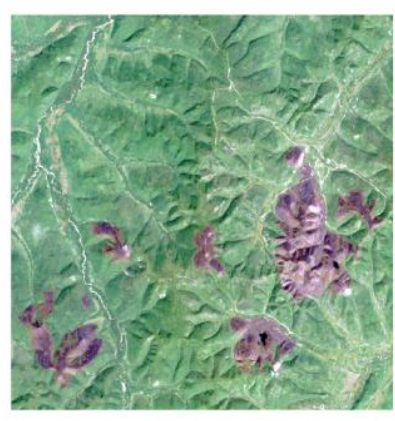

(a)

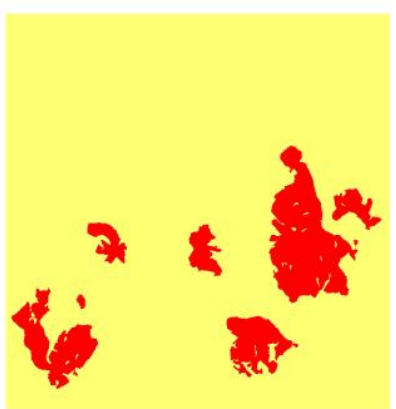

(b)

Figure 4. Landsat image after fire and its ground truth map: (a) post-fire image; (b) ground truth map.

\subsection{Accuracy Analysis of Burned Area Mapping}

Figure 5 shows the mapping results of the burned areas produced by different methods. It can be seen from Figure 5 that compared with the reference map shown in Figure 4(b), all methods can identify the main burned areas. However, there are obvious false detections in the burned area maps produced by different methods based on the NBR (Figure 5(a) (c)), such as the area marked by the ellipse in Figure 5, while there are obvious missed detections in the burned area maps produced from the BAI (Figure 5(d) (f)), such as the area marked by the rectangle in Figure 5.

Table 1 is the result of quantitative accuracy evaluation of burned area maps produced by different methods using OA, KC, FA, and MA. It can be seen from Table 1 that regardless of whether NBR or BAI is used for burned area mapping, the OA and $\mathrm{KC}$ of the proposed method are higher than other methods to varying degrees. This shows that the overall mapping performance of the proposed method is better than other methods, and the burned area map generated by the proposed method has the highest spatial consistency with the real reference map. At the same time, Table 1 also shows that whether using NBR or BAI for burned area mapping, the MA of the proposed method is significantly lower than that of other methods. Unfortunately, compared with other methods, the FA of the proposed method is higher. In addition, it is not difficult to find from Table 1 that no matter which method is used for burned area mapping, the accuracy of NBR-based burned area mapping is better than BAI, and the reason for the poor mapping accuracy of BAI is that the MA of the BAI-based burned area mapping results is too high. 


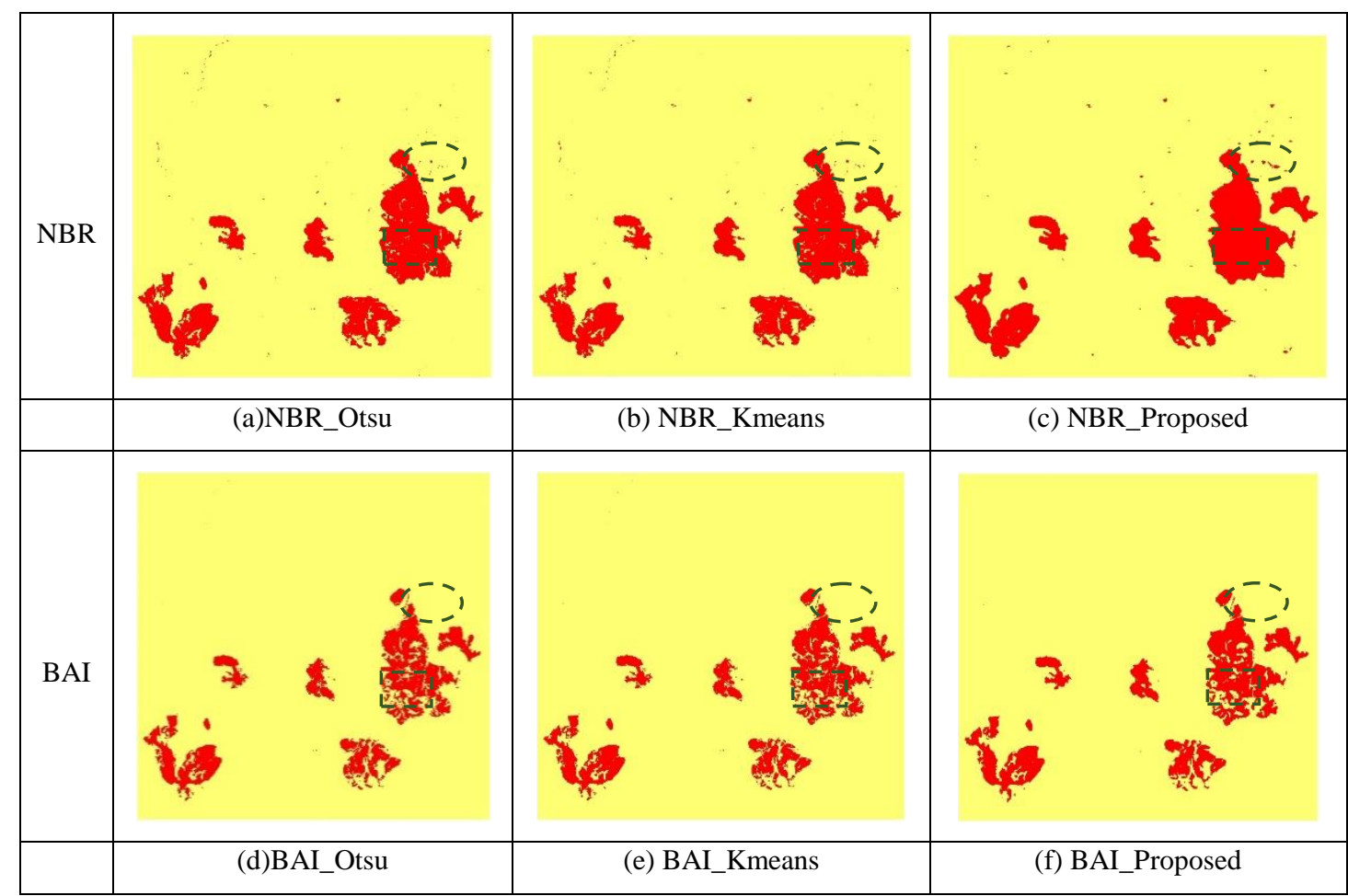

Figure 5. Burned area mapping results of different methods: (a) (c): NBR-based burned area mapping results by Otsu, Kmeans and Proposed method; (d) (f): BAI-based burned area mapping results by Otsu, Kmeans and Proposed method.

\begin{tabular}{|l|l|l|l|l|l|l|}
\hline & \multicolumn{3}{|c|}{ NBR } & \multicolumn{3}{c|}{ BAI } \\
\hline Indexes & NBR_Otsu & NBR_Kmeans & NBR_Proposed & BAI_Otsu & BAI_Kmeans & BAI_Proposed \\
\hline OA & $98.81 \%$ & $98.80 \%$ & $\mathbf{9 9 . 0 2 \%}$ & $96.90 \%$ & $96.90 \%$ & 97.19\% \\
\hline KC & $93.48 \%$ & $93.64 \%$ & $\mathbf{9 4 . 8 2 \%}$ & $81.00 \%$ & $81.00 \%$ & 82.94\% \\
\hline FA & $3.83 \%$ & $3.81 \%$ & $6.73 \%$ & $0.98 \%$ & $0.98 \%$ & $1.00 \%$ \\
\hline MA & $7.80 \%$ & $7.85 \%$ & $\mathbf{2 . 4 4 \%}$ & $29.08 \%$ & $29.08 \%$ & 26.37\% \\
\hline
\end{tabular}

Table 1. Quantitative evaluation results of mapping accuracy of different methods

\subsection{Mapping Performance Analysis of Different Methods on Uncertain Pixels}

As mentioned earlier, in the fire index map, those uncertain pixels with medium index values are often the most difficult to be correctly classified. These uncertain pixels are a major source of error in the mapping of the burned areas. The ability to correctly classify these uncertain pixels directly reflects the mapping performance of different methods. To this end, this subsection compares and analyzes the classification capabilities of different methods on uncertain pixels. Table 2 shows the OA of different methods on certain or uncertain pixels. It can be seen from Table 2 that regardless of NBR or BAI, the mapping accuracy of different methods on the certain pixels is significantly better than that on the uncertain pixels. Moreover, the mapping accuracy of the proposed method is significantly better than other methods on uncertain pixels, especially when the BAI is used to map the burned areas. For certain pixels, although the proposed method has the highest mapping accuracy, the difference in mapping accuracy of different methods is very small. This result shows that the proposed method is effective and has certain advantages in accurately distinguishing the classes of uncertain pixels. And from Table 2, it can be found that the different methods have relatively small differences in the mapping accuracy on the certain pixels of NBR and BAI, but the differences in the mapping accuracy on their uncertain pixels are very obvious. This shows that the reason why NBR's burned area mapping ability is better than $\mathrm{BAI}$ is mainly due to the distinguishability of uncertain pixels.

\begin{tabular}{|l|l|l|l|l|}
\hline \multirow{2}{*}{} & \multicolumn{2}{|c|}{ NBR } & \multicolumn{2}{c|}{ BAI } \\
\cline { 2 - 5 } & $\begin{array}{l}\text { Certain } \\
\text { Pixels }\end{array}$ & $\begin{array}{l}\text { Uncertain } \\
\text { Pixels }\end{array}$ & $\begin{array}{l}\text { Certain } \\
\text { Pixels }\end{array}$ & $\begin{array}{l}\text { Uncertain } \\
\text { Pixels }\end{array}$ \\
\hline Otsu & $99.632 \%$ & $94.811 \%$ & $98.144 \%$ & $66.179 \%$ \\
\hline Kmeans & $99.631 \%$ & $94.798 \%$ & $98.144 \%$ & $66.174 \%$ \\
\hline Proposed & $\mathbf{9 9 . 6 8 0 \%}$ & $\mathbf{9 5 . 7 9 5 \%}$ & $\mathbf{9 8 . 2 0 5 \%}$ & $\mathbf{7 1 . 8 4 6 \%}$ \\
\hline
\end{tabular}

Table 2. OA of different methods on certain or uncertain pixels

\section{CONCLUSIONS}

The Considering that there is still room for improvement in the accuracy of current burned area mapping based on single-date remote sensing image, this paper proposes an unsupervised forest burned area mapping method based on fire index enhancement and GRNN model. The proposed method firstly integrates spatial context information in an adaptive manner to enhance the existing fire index to improve its ability to indicate the burned areas, and then uses the modified GRNN model that considers the spatial correlation between pixels in the image to 
binarize the enhanced fire index to generate the final burned area map. The experimental results of burning zone mapping in a forest area in Inner Mongolia, China demonstrate the effectiveness and superiority of the proposed method. In particular, the proposed method has certain advantages in correctly classifying uncertain pixels. In addition, the proposed method uses certain pixels generated by uncertainty analysis as training samples for GRNN model training instead of a large number of artificially labeled samples. Therefore, the proposed method is unsupervised, which will enhance its practical application value.

\section{ACKNOWLEDGEMENTS}

This paper was supported by the National Key Research and Development Program of China under Grant 2018YFF0215006 and The Project Supported by the Open Fund of Key Laboratory of Urban Land Resources Monitoring and Simulation, Ministry of Land and Resources under Grant KF2019-04-046.

\section{REFERENCES}

Bastarrika, A., Chuvieco, E., Martín, M.P., 2011. Mapping burned areas from Landsat TM/ETM+ data with a two-phase algorithm: Balancing omission and commission errors. Remote Sensing of Environment 115, 1003-1012.

Chuvieco, E., Martín, M.P., Palacios, A., 2002. Assessment of different spectral indices in the red-near-infrared spectral domain for burned land discrimination. Int J Remote Sens 23, 5103-5110.

Engelbrecht, J., Theron, A., Vhengani, L., Kemp, J., 2017. A Simple Normalized Difference Approach to Burnt Area Mapping Using Multi-Polarisation C-Band SAR. Remote SensBasel 9.

Havens, T.C., Bezdek, J.C., Leckie, C., Hall, L.O., Palaniswami, M., 2012. Fuzzy c-Means Algorithms for Very Large Data. IEEE Transactions on Fuzzy Systems 20, 1130-1146.

Kouhi, A., Seyedarabi, H., Aghagolzadeh, A., 2020. Robust FCM clustering algorithm with combined spatial constraint and membership matrix local information for brain MRI segmentation. Expert Systems with Applications 146, 113159.

Li, C., Bovik, A.C., Wu, X., 2011. Blind Image Quality Assessment Using a General Regression Neural Network. IEEE Transactions on Neural Networks 22, 793-799.

Li, L., Xu, T., Chen, Y., 2016. Improved Urban Flooding Mapping from Remote Sensing Images Using Generalized Regression Neural Network-Based Super-Resolution Algorithm. Remote Sens-Basel 8.

Liu, S., Zheng, Y., Dalponte, M., Tong, X., 2020. A novel fire index-based burned area change detection approach using Landsat-8 OLI data. European Journal of Remote Sensing 53, 104-112.

Pulvirenti, L., Squicciarino, G., Fiori, E., Fiorucci, P., Ferraris, L., Negro, D., Gollini, A., Severino, M., Puca, S., 2020. An Automatic Processing Chain for Near Real-Time Mapping of Burned Forest Areas Using Sentinel-2 Data. Remote Sens-Basel 12.
Roteta, E., Bastarrika, A., Padilla, M., Storm, T., Chuvieco, E., 2019. Development of a Sentinel-2 burned area algorithm: Generation of a small fire database for sub-Saharan Africa. Remote Sensing of Environment 222, 1-17.

Roy, D.P., Huang, H., Boschetti, L., Giglio, L., Yan, L., Zhang, H.H., Li, Z., 2019. Landsat-8 and Sentinel-2 burned area mapping - A combined sensor multi-temporal change detection approach. Remote Sensing of Environment 231, 111254.

Veraverbeke, S., Harris, S., Hook, S., 2011. Evaluating spectral indices for burned area discrimination using MODIS/ASTER (MASTER) airborne simulator data. Remote Sensing of Environment 115, 2702-2709.

Woźniak, E., Aleksandrowicz, S., 2019. Self-Adjusting Thresholding for Burnt Area Detection Based on Optical Images. Remote Sens-Basel 11. 